\title{
Diagnostic Method Combining the Lookup Tables and Fault Models Applied on a Hybrid Electric Vehicle
}

Christofer Sundström, Erik Frisk and Lars Nielsen

\section{Linköping University Post Print}

\section{Tweet}

N.B.: When citing this work, cite the original article.

(C2016 IEEE. Personal use of this material is permitted. However, permission to reprint/republish this material for advertising or promotional purposes or for creating new collective works for resale or redistribution to servers or lists, or to reuse any copyrighted component of this work in other works must be obtained from the IEEE.

Christofer Sundström, Erik Frisk and Lars Nielsen, Diagnostic Method Combining the Lookup Tables and Fault Models Applied on a Hybrid Electric Vehicle, 2016, IEEE Transactions on Control Systems Technology, (24), 3, 1109-1117.

http://dx.doi.org/10.1109/TCST.2015.2480008

Postprint available at: Linköping University Electronic Press

http://urn.kb.se/resolve?urn=urn:nbn:se:liu:diva-128736 


\title{
Diagnostic Method Combining Look-up Tables and Fault Models Applied on a Hybrid Electric Vehicle
}

\author{
Christofer Sundström, Erik Frisk, and Lars Nielsen
}

\begin{abstract}
A common situation in industry is to store measurements for different operating points in look-up tables, often called maps. They are used in many tasks, e.g. in control and estimation, and therefore considerable investments in engineering time are spent in measuring them which usually make them accurate descriptions of the fault free system. They are thus well suited for fault detection, but, however, such a model can not give fault isolation since only the fault free behavior is modeled. One way to handle this situation would be to also map all fault cases but that would require measurements for all faulty cases, which would be costly if at all possible. Instead, the main contribution here is a method to combine the look-up model with analytical fault models. This makes good use of all modeling efforts of the look-up model for the fault free case, and combines it with fault models with reasonable modeling and calibration efforts, thus decreasing the engineering effort in the diagnosis design. The approach is exemplified by designing a diagnosis system monitoring the power electronics and the electric machine in a hybrid electric vehicle. An extensive simulation study clearly shows that the approach achieves both good fault detectability and isolability performance. A main point is that this is achieved without the need for neither measurements of a faulty system nor detailed physical modeling, thus saving considerable amounts of development time.
\end{abstract}

\section{INTRODUCTION}

Fault monitoring and diagnosis is used to detect and isolate faults in a system. The importance of monitoring vehicle powertrains increases with the vehicle complexity, and in [1] diagnosis in the vehicle application is described. There are data driven diagnostic approaches available [2], [3], but model based approaches [4] are typically preferred in the automotive industry. Examples of such methods from the artificial intelligence field is consistency based diagnosis [5], [6], [7], and from the control community there are techniques like parity equations [8], variable elimination [9], parameter estimation [10], stateobservers [11], [12], and residual generators [13].

A model based diagnosis system checks the consistency between observations and a model of the system to be monitored. The models are developed to different level of detail, and a common approach in the automotive industry is to use models including a look-up table containing measurement data. These models are commonly called map based models, and are directly calibrated from measurements of the non-faulty case. In general these models are motivated by many tasks other than diagnosis, and therefore considerable investments are made in them. They are obtained after substantial calibration so they accurately describe the outputs of the fault free system, which directly results in good fault detection performance. However,

C. Sundström, E. Frisk, and L. Nielsen are with the Dept. of Electrical Engineering, Linköping University, SE-581 83 Linköping, Sweden $\{$ csu,frisk,lars\}@ isy.liu.se one drawback with a map based model is the difficulty to isolate faults from each other since internal physical phenomena are not described by the model. One way to achieve fault isolability using a map based model is to collect data when the faults have occurred in the system to be monitored, which is a costly solution due to the many fault cases. Another approach to achieve good fault isolability is to use models that explicitly describe how different faults affect the system to be monitored. Depending on the fault mode, such models give different reactions in behavior, both qualitatively and quantitatively, and can therefore be used to separate faults from each other. To achieve good diagnostic performance only using a model explicitly including the faults, the model needs to be accurate including detailed physical modeling, which also is costly.

\section{A. Main idea and contributions}

The main idea here is an approach to combine a carefully measured look-up model for the fault free case with analytical fault models that explicitly describe how the faults affect the monitored system. The main goal is to achieve both good fault detection and isolation performance with much less calibration and engineering time compared to previous methods. It is a way of making good use of all effort in calibrating the map based model, and, further, compared to only using a model explicitly describing the faults, less accurate models that explicitly describe the faults can be used in the diagnosis system based on a combination of models. Compared to a preliminary version of the idea presented in [14], the concepts have been clarified and generalized, but the most important extension is in the analysis and simulation study. The approach is applied to the design of a diagnosis system monitoring the power electronics and the electric machine used in a hybrid electric vehicle (HEV), where safety, component protection, and high up-time of the vehicle are important. Evaluating the new diagnostic approach, it is demonstrated that a main benefit of the approach is that measurements of a faulty system is not needed to achieve fault isolability. It is also demonstrated that the approach decreases the accuracy demands on the model used for fault modeling compared to designing a diagnosis system without using the map based model.

\section{B. Outline}

The models used in the diagnosis design of the electric machine are described in Section II, and in Section III these models are combined to include fault models in the map based model. The engineering value of using this combined model in a diagnosis system is evaluated in Section $\mathrm{V}$, and finally the conclusions are given in Section VI. 


\section{Models OF THE ELECTRIC MACHINE}

In HEVs mainly permanent magnet synchronous machines (PMSM) are used since this type of machine in general has higher efficiency compared to other machine types [15], [16]. In this section two models of a PMSM are presented, that later are used to illustrate the approach in the design of the diagnosis system. The first model includes a map that describes the power losses in the machine and is presented in Section II-A. To model how faults affect the machine and power electronics, which is not captured in the map based model, the second model is based on analytical expressions and is presented in Section II-B. Both these models are static since the dynamics of the electric machine is considered fast compared to the time scales used in the diagnosis systems, but the diagnostic approach can be applied to dynamic systems as well.

\section{A. Map based model}

The map based model describes the power losses in the machine and the power electronics, and is based on measurements to find the difference between the electrical and mechanical powers. The inputs to the map of the power losses, $P_{e m, l}^{m a p}$ is the delivered torque, $T_{e m}$, motor speed, $\omega_{e m}$, and battery voltage, $U_{b}$, as

$$
P_{e m, l}^{m a p}=f\left(T_{e m}, \omega_{e m}, U_{b}\right)
$$

and the power losses are given in Figure 1. There are limitations in the delivered torque from the machine, denoted $T_{e m, \min }$ in generator mode and $T_{e m, \max }$ in motor mode, that are functions of $\omega_{e m}$ and $U_{b}$. The delivered torque, $T_{e m}$, is modeled to be equal to the requested torque, $T_{e m, r e q}$, if the requested torque is within the limitations of what the machine is able to deliver

$$
T_{e m}^{\text {map }}= \begin{cases}T_{e m, \text { min }}, & T_{e m, r e q}<T_{e m, \text { min }} \\ T_{e m, r e q}, & T_{e m, \text { min }} \leq T_{e m, r e q}<T_{e m, \max } \\ T_{e m, \text { max }}, & T_{e m, r e q} \geq T_{e m, \text { max }}\end{cases}
$$

The mechanical power delivered by the machine, $P_{e m, m}$, is used to calculate the electrical power

$$
P_{e m, e}=P_{e m, m}+P_{e m, l}^{m a p}=T_{e m}^{m a p} \omega_{e m}+P_{e m, l}^{m a p}
$$

The power electronics is included in the model and is assumed to be an ideal component. The battery current, $I_{b}$, is computed by dividing $P_{e m, e}$ with the battery terminal voltage, $U_{b}$

$$
I_{b}=\frac{P_{e m, e}}{U_{b}}
$$

\section{B. Analytical model}

A PMSM can be modeled as a separately excited DC motor with constant field [17], since the stator of a PMSM consists of windings, and the armature of permanent magnets. This is done in the model based on analytical expressions, where the resistive and frictional losses are modeled to represent the losses of the machine. The torque is modeled to be proportional to the current, $I_{e m}$, except for the frictional losses that are modeled to be proportional to $\omega_{e m}$ [18]

$$
T_{e m}^{a}=k I_{e m}-c_{f} \omega_{e m}
$$

where $c_{f}$ is a friction constant and $k$ is a machine constant. The current is calculated using the voltage, $U_{e m}$, supplied by

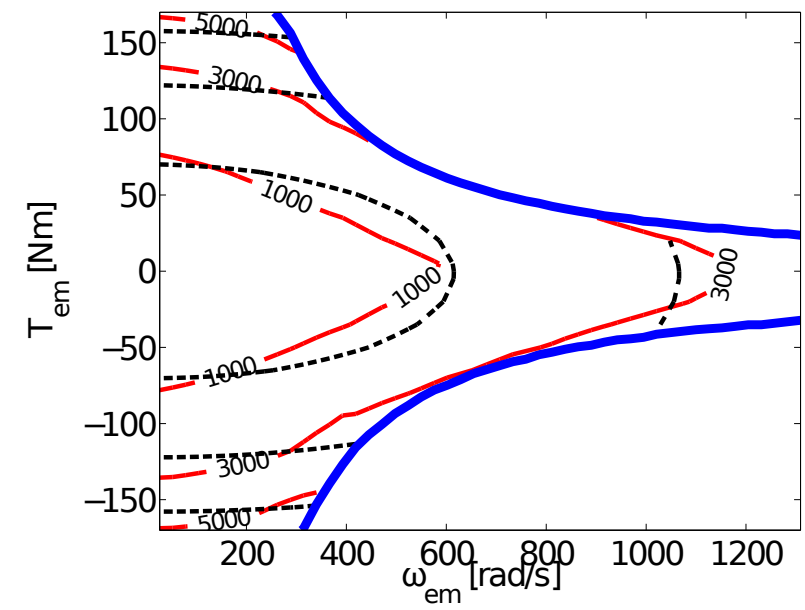

Fig. 1. The power losses [W] of the machine and power electronics. The solid (thin) red lines show the measured losses in the map described in Section II-A, the dashed lines the losses in the model described in Section II-B, and the solid (thick) blue line the torque limitation of the machine.

the power electronics and the electromotive force, that depends on the speed of the machine, $\omega_{e m}$, as $k \omega_{e m}$

$$
I_{e m}=\frac{1}{R_{e m}}\left(U_{e m}-k \omega_{e m}\right)
$$

where $R_{e m}$ is the resistance in the electric machine. The power losses in the machine are computed using

$$
P_{e m, l}^{a}=U_{e m} I_{e m}-T_{e m}^{a} \omega_{e m}
$$

Substituting $U_{e m}$ and $I_{e m}$ from (5) and (6) gives

$$
P_{e m, l}^{a}=R_{e m}\left(\frac{\left(T_{e m}^{a}\right)^{2}}{k^{2}}+\frac{2 c_{f}}{k^{2}} \omega_{e m} T_{e m}^{a}+\frac{c_{f}^{2}}{k^{2}} \omega_{e m}^{2}\right)+c_{f} \omega_{e m}^{2}
$$

This model is fitted to the measured losses used in the map based model. The parameters in the analytical model are found by minimizing the least square error between (1) and (8), and the parameters $k, R_{e m}$, and $c_{f}$ are found to be $0.50 \mathrm{Nm} / \mathrm{A}$, $0.065 \Omega$, and $0.0029 \mathrm{Nm} / \mathrm{s}$, respectively. The battery voltage is assumed to be the open circuit voltage, i.e. $250 \mathrm{~V}$, when using the map to find the losses. The power losses computed in (8) are compared with the measured losses in Figure 1.

The power electronics is assumed to be an ideal component also in this model, and the battery current is described by

$$
I_{b}=\frac{I_{e m} U_{e m}}{U_{b}}
$$

1) Controller: The energy management operating on vehicle level requests a torque, $T_{e m, r e q}$, from the electric machine. The machine controller computes a requested voltage, $U_{e m}^{c t r l}$, from the power electronics in order for the machine to, if possible, deliver the requested torque. The controller is an open loop controller and $U_{e m}^{c t r l}$ is computed by

$$
U_{e m}^{c t r l}=\left(\frac{T_{e m, r e q}}{k}+\frac{c_{f}}{k} \omega_{e m}\right) R_{e m}+k \omega_{e m}
$$

The model for the power electronics supplies this voltage to the machine when the component is fault free, i.e.

$$
U_{e m}=U_{e m}^{c t r l}
$$




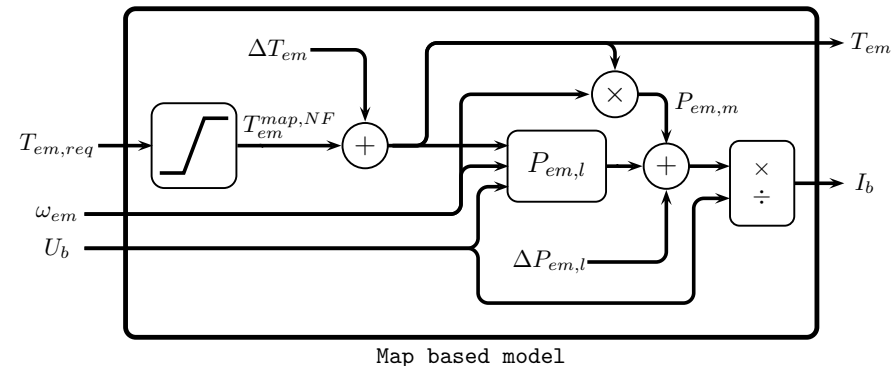

Fig. 2. Schematic structure of the map based model extended with $\Delta T_{e m}$ and $\Delta P_{e m, l}$ to include the possibility to model faults in the machine.

\section{COMBINING MAP WITH ANALYTICAL FAULT MODELS}

As stated above, the map based model presented in Section II-A is beneficial to model the nominal behavior of the machine due to its high accuracy. However, the model has the disadvantage that the parameters affected when a fault has occurred are not explicitly included in the model. In the fault free case, the map based model of the machine delivers the requested torque, as long as the machine is capable of delivering the torque, as can be seen in the schematic structure of the model in Figure 2. The battery current, $I_{b}$, is calculated using the mechanical power, $P_{e m, m}$, and the power losses, $P_{e m, l}$, that is a map and depends on the operating points of the machine, as described in Section II-A. The superscript $N F$ indicates a non-faulty signal, e.g. $T_{e m}^{\operatorname{map}, N F}$ is the nominal delivered torque computed by (2).

The two main ways to model faults in a map based model is to modify the input or output signals of the map. Here a fault affecting the delivered torque is modeled to affect the input of the map using $\Delta T_{e m}$ according to Figure 2. This results in that the power losses changes when there is a fault affecting the delivered torque. A fault directly affecting the machine power losses affects the output of the map, i.e. the battery current, and is modeled using $\Delta P_{e m, l}$. Expressions for $\Delta T_{e m}$ and $\Delta P_{e m, l}$ are derived in Sections III-A and III-B respectively.

Three fault modes are considered in the design of the diagnosis systems described in Section V, and these faults are also used to evaluate the diagnosis system in simulations. Two of the faults affect the electric machine, by modifications in the resistance of the machine and the lumped torque and speed constant $k$ used in the analytical model. A fault in the power electronics is modeled to result in that the voltage on the electric machine is not the requested voltage. The faults are modeled as

$$
\begin{aligned}
k & =k^{n o m}\left(1+f_{e m, k}\right) \\
R_{e m} & =R_{e m}^{n o m}\left(1+f_{e m, R}\right) \\
U_{e m} & =U_{e m}^{c t r l}\left(1+f_{p e}\right)
\end{aligned}
$$

where $k^{n o m}$ and $R_{e m}^{n o m}$ are the nominal values of the parameters and according to (11) $U_{e m}=U_{e m}^{c t r l}$ in the fault free case. Note that the faults in (12) are examples of how a fault in the electric machine and power electronics can be represented in the model. However, the selected faults are important to monitor since they affect the delivered torque from the electric machine, as can be seen by combining (5) and (6) for the analytical model

$$
T_{e m}^{a}=k\left(\frac{U_{e m}}{R_{e m}}-\frac{k}{R_{e m}} \omega_{e m}\right)-c_{f} \omega_{e m}
$$

In the analytical model of the electric machine described in Section II-B, it is straightforward to induce the faults described in (12) since these parameters are included in the model. The accuracy is however generally lower in this category of models compared to the map based model. Therefore, the map based model is used to model the fault free case, and the analytical model is used to model the influence of the faults on the electrical machine to achieve both good fault detection and fault isolation performance.

\section{A. Finding an expression for $\Delta T_{e m}$}

From (13) it is seen that all three fault modes in (12) affect the delivered torque of the electric machine, and is here modeled according to

$$
T_{e m}=T_{e m}^{m a p, N F}+\Delta T_{e m}
$$

where $\Delta T_{e m}$ is the difference between $T_{e m}^{r e q}$ and $T_{e m}$ due to a fault in the system. To find the expression for $\Delta T_{e m}$, the torque delivered by the faulty machine is computed using (13), and the delivered torque in the fault free case, $T_{e m}^{a, N F}$, is also computed using (13), but with the nominal values of the parameters in the machine. The parameters $k$ and $R_{e m}$, and the voltage $U_{e m}$ used to calculate $T_{e m}^{a}$, include models for the faults according to (12), and $\Delta T_{e m}$ is expressed by

$$
\begin{aligned}
& \Delta T_{e m}=T_{e m}^{a}-T_{e m}^{a, N F} \\
= & \frac{k}{R_{e m}}\left(U_{e m}-k \omega_{e m}\right)-\frac{k^{n o m}}{R_{e m}^{n o m}}\left(U_{e m}^{N F}-k^{n o m} \omega_{e m}\right)
\end{aligned}
$$

The voltages $U_{e m}^{N F}$ and $U_{e m}$ are computed by (11) and (12c), respectively, where $U_{e m}^{c t r l}$ is used. The voltage $U_{e m}^{c t r l}$ is not modeled in the map based model, and is therefore computed using the controller in the analytical model given in (10) using the limited requested torque $T_{e m}^{m a p, N F}$ computed by (2). Information about if the system is faulty or fault free is not assumed to be known in the controller of the machine where $U_{e m}^{c t r l}$ is set, and therefore $R_{e m}$ and $k$ in (10) are the nominal values even if there is a fault in the machine affecting these parameters. The expression for $U_{e m}^{c t r l}$ used to compute $\Delta T_{e m}$ and $\Delta P_{e m, l}$ is thereby

$$
U_{e m}^{c t r l}=\left(\frac{T_{e m}^{m a p, N F}}{k^{n o m}}+\frac{c_{f}}{k^{n o m}} \omega_{e m}\right) R_{e m}^{n o m}+k^{n o m} \omega_{e m}
$$

\section{B. Finding an expression for $\Delta P_{e m, l}$}

The expression for the power losses in the analytical model is given in (8), and the expression states that $f_{e m, k}$ and $f_{e m, R}$ affect the power losses in the model. The losses also considering the faulty cases are modeled as

$$
P_{e m, l}=P_{e m, l}^{m a p, N F}+\Delta P_{e m, l}
$$

where $P_{e m, l}^{\operatorname{map}, N F}$ is the nominal map from (1), and $\Delta P_{e m, l}$ describes the difference in the power losses in the machine in the fault free and faulty cases of the machine. The losses 
in the faulty case are computed by (8), and the losses in the fault free case, $P_{e m, l}^{a, N F}$, are also computed by (8), but with the nominal values of the parameters $R_{e m}$ and $k$. The torque used in the computations of $P_{e m, l}^{a}$ and $P_{e m, l}^{a, N F}$ is the delivered torque $T_{e m}$ from the machine in the map based model, see Figure 2. The modifications in the power losses is computed by

$$
\begin{aligned}
& \Delta P_{e m, l}=P_{e m, l}^{a}-P_{e m, l}^{a, N F} \\
& \quad=\left(\frac{R_{e m}}{k^{2}}-\frac{R_{e m}^{n o m}}{\left(k^{n o m}\right)^{2}}\right)\left(T_{e m}^{2}+2 c_{f} \omega_{e m} T_{e m}+c_{f}^{2} \omega_{e m}^{2}\right)
\end{aligned}
$$

\section{ISOLABILITY GAIN BY COMBINING MODELS}

In this section the maximum theoretical isolability performance of a diagnosis system based on the map based model is discussed. A single-fault assumption is made in the diagnosis system design since the probability for a fault to occur is low in the automotive application. The theoretical isolability performance using only the map based model is compared with the performance when combining the map based model with the fault models obtained in Section III.

\section{A. Theoretical fault isolability using map based model}

First, consider the case when using only the map based model, without any fault models. There are three fault modes to be monitored, see (12). On the basis of using only the map based model, it is reasonable that all three faults affect the delivered torque

$$
T_{e m}=g_{1}\left(f_{e m, k}, f_{e m, R}, f_{p e}\right)
$$

while the power losses only depend on the fault modes in the electric machine since the power electronics is modeled as an ideal component.

$$
P_{e m, l}=g_{2}\left(f_{e m, k}, f_{e m, R}\right)
$$

Note that $g_{1}$ and $g_{2}$ also depend on other variables.

Based on these two equations it is obvious that it is not possible to achieve full fault isolability, e.g. since both $f_{e m, k}$ and $f_{e m, R}$ affects both equations and no information about how the faults affect the system is available.

\section{B. Theoretical fault isolability using a combined model}

Now consider the case where fault models are used in combination with the map. As stated in Section IV-A, fault models are required to isolate the fault modes from each other in the diagnosis system. Here the faults' influence on $\Delta T_{e m}$ and $\Delta P_{e m, l}$ described in (15) and (18) are used in the diagnosis system. It is assumed that the faults are constant or slowly varying, and this is modeled as $\dot{f}=0$. Note that the parameters $k$ and $R_{e m}$, and the voltage $U_{e m}$ all are included in the expression for $T_{e m}$, and that the faults affect the torque in different ways. This means that full fault isolability can be achieved using only information about how $T_{e m}$ is modified i.e. by only using (12) and (15). The information from how the faults affect $\Delta P_{e m, l}$ is however also used in the estimation of the faults using observers in the next section.

\section{DESIGN OF A DIAGNOSIS SYSTEM}

To evaluate the influence of the new approach on the diagnosis performance, residual generators are designed based on 1) the combined model and 2) only the analytical model. The residual generation approach used in the evaluation is a state-observer design technique.

\section{A. State space formulation of the model}

The model considered below is the combined model of the electric machine. The model is static since the dynamics of the electric machine is fast compared to the time scales considered here. In the model the faults are included as states, $x_{1}$, that are estimated in the observers. The model is given in the form

$$
\begin{aligned}
x_{1, t} & =x_{1, t-1}+\omega_{t} \\
x_{2, t} & =G\left(x_{1, t}, u_{t}\right) \\
y_{t} & =h\left(x_{1, t}, x_{2, t}, u_{t}\right)+\nu_{t}
\end{aligned}
$$

where $x_{2}$ is the vector of algebraic variables, $u$ is the vector of known input signals, $\omega$ is the uncertainty in the faults to be estimated, $\nu$ is the measurement noise, and the subscript $t$ is the time index. The algebraic variables $x_{2}$ and $G\left(x_{1}, u\right)$ are from (1), (2), (3), (4), (11), (12), (14), (15), (16), and (18) given by

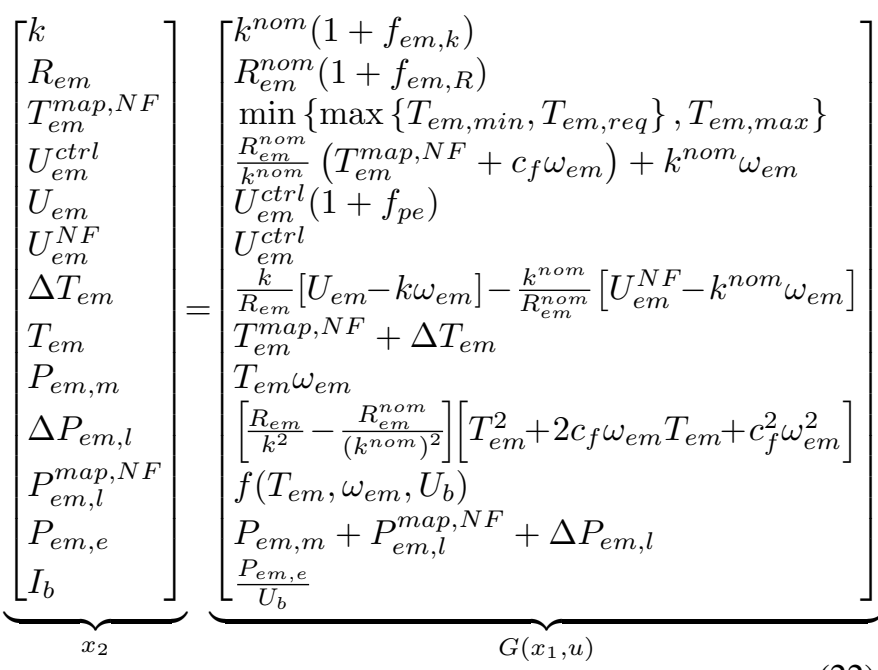

The input signals of the model are the requested torque, $T_{e m, r e q}$, angular speed, $\omega_{e m}$, and the battery voltage $U_{b}$

$$
u=\left[\begin{array}{lll}
T_{e m, r e q} & \omega_{e m} & U_{b}
\end{array}\right]^{T}
$$

The output signals are the delivered torque, $T_{e m}$, and the battery current, $I_{b}$, that are calculated in (21c) and are given by

$$
h\left(x_{1}, x_{2}, u\right)=\left[T_{e m} I_{b}\right]^{T}
$$

The torque can be estimated from other sensors and an extended model [19], but here a torque sensor is used for simplicity.

\section{B. Fault estimation}

The designed diagnosis systems are based on state-observers estimating the considered faults in (12). A direct way is to estimate all three faults in one single observer. Several approaches are available and here an Extended Kalman Filter 

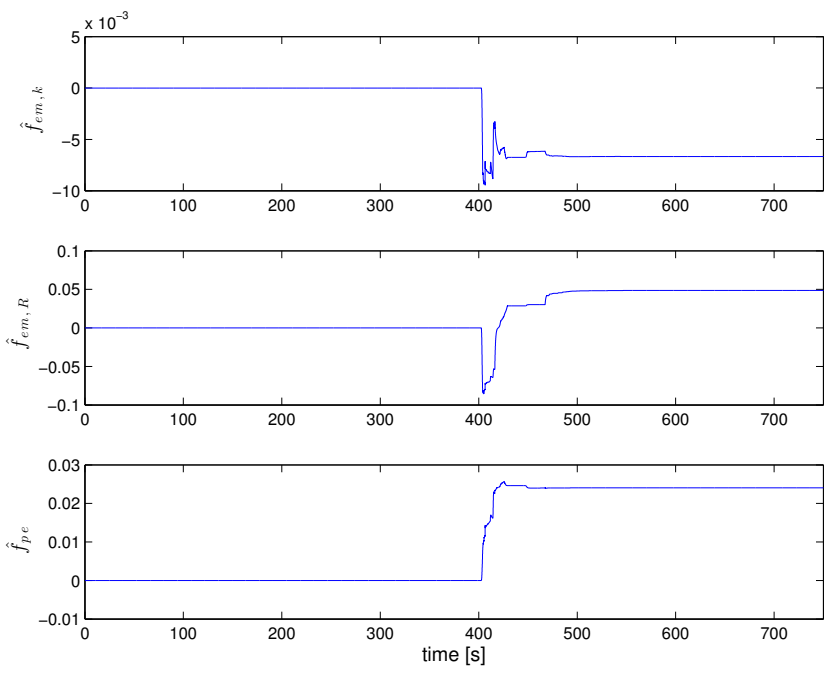

Fig. 3. Estimated faults when $k=0.97 k_{n o m}$ after 400 seconds using $\mathcal{O}_{\text {all }}$.

(EKF) [20] is used. A constant gain observer is not applicable due to that the sign of the feedback gain parameter, $K$, to ensure a stable observer depends on the operating point of the system. The observer denoted as $\mathcal{O}_{\text {all }}$ is using the model equations given by (22), and is described by

$$
\mathcal{O}_{\text {all }}:\left\{\begin{array}{l}
\hat{x}_{1, t+1}=\hat{x}_{1, t}+K_{t}\left(y_{t}-\hat{y}_{t}\right) \\
\hat{y}_{t}=h\left(\hat{x}_{1, t}, G\left(\hat{x}_{1, t}, u_{t}\right), u_{t}\right)
\end{array}\right.
$$

where $x_{1}=\left[\begin{array}{lll}f_{e m, k} & f_{e m, R} & f_{p e}\end{array}\right]^{T}$. These states are observable since the faults affect the output signals in different ways, see the model in (22) and (24). The measurement noise in (21c) is modeled as Gaussian distributed white noise and is denoted as $\nu_{T_{e m}}$ and $\nu_{I_{b}}$ respectively

$$
y=\left[\begin{array}{l}
T_{\text {em,sens }} \\
I_{b, \text { sens }}
\end{array}\right]=\left[\begin{array}{l}
T_{e m}+\nu_{T_{e m}} \\
I_{b}+\nu_{I_{b}}
\end{array}\right]
$$

To evaluate the designed observer, the faults in (12) are induced one by one in the vehicle model and the observer estimates the faults. The gain parameter $K_{t}$ is computed in each time step using the covariance matrices for the measurement noise $R$ and the process noise $Q$. The faults are induced as a step after 400 seconds in the simulations, and the sizes of the induced faults are $f_{e m, k}=-0.03, f_{e m, R}=-0.03$, and $f_{p e}=-0.01$. The driving cycle used is FTP75 and the estimated faults when $f_{e m, k}$ has occurred, i.e. $x_{1}=\left[\begin{array}{lll}-0.03 & 0 & 0\end{array}\right]^{T}$, are presented in Figure 3. As can be seen in the figure, all faults are nonzero after the fault is induced, and the estimated faults have converged to almost constant values that are $\hat{x}_{1}=\left\{\hat{f}_{e m, k}, \hat{f}_{e m, R}, \hat{f}_{p e}\right\}=$ $\{-0.0067,0.049,0.024\}$. This is significantly separate from the true values, $x_{1}^{\star}=\left[\begin{array}{lll}-0.03 & 0 & 0\end{array}\right]^{T}$, and to understand the reason for this, the outputs $T_{e m}$ and $I_{b}$ for the two cases $x_{1}=\hat{x}_{1}$ and $x_{1}=x_{1}^{\star}$ are investigated.

The output signals based on $\hat{x}_{1}$ and $x_{1}^{\star}$ are denoted as $\hat{T}_{e m}, \hat{I}_{b}$, and $T_{e m}^{\star}, I_{b}^{\star}$, respectively, and the differences between the outputs are computed as

$$
\Delta_{T_{e m}}=\hat{T}_{e m}-T_{e m}^{\star}, \Delta_{I_{b}}=\hat{I}_{b}-I_{b}^{\star}
$$

The maximum magnitudes of $\Delta_{T_{e m}}$ and $\Delta_{I_{b}}$ in the simulation are $0.002 \mathrm{Nm}$ and $0.01 \mathrm{~A}$ respectively, which corresponds to
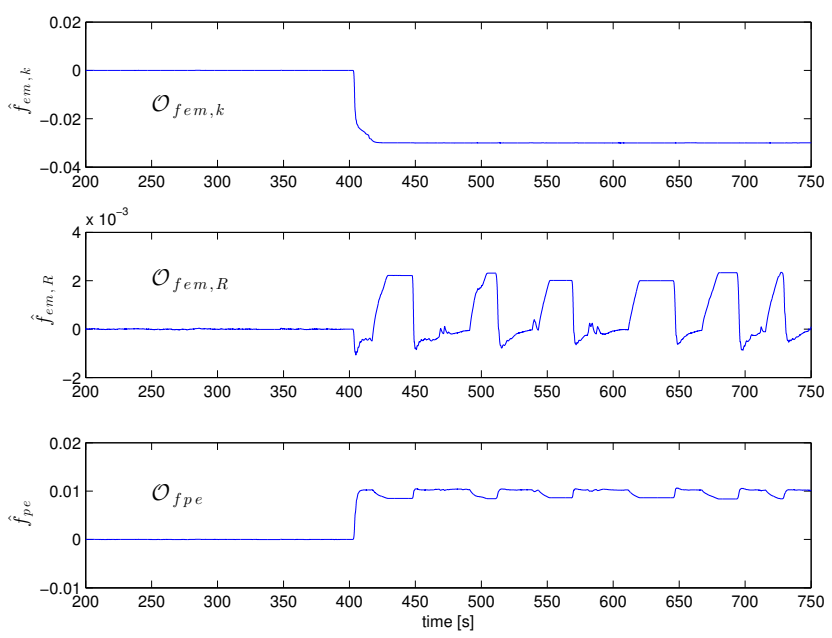

Fig. 4. The estimated faults in the three observers $\mathcal{O}_{e m, k}, \mathcal{O}_{e m, R}$, and $\mathcal{O}_{p e}$ when $k=0.97 k^{n o m}$.

as small values as $0.01 \%$ of the maximum magnitude of both output signals. A conclusion of this is that even though the model is observable, the sensitivity is small and thereby the estimation is difficult in practice.

1) Three observers estimating one fault each: To overcome the observability difficulty using one observer, the single-fault assumption is utilized and three observers are designed to estimate one fault each. In each observer it is assumed that the other two faults are zero, and the three observers estimating $f_{e m, k}, f_{e m, R}$, and $f_{p e}$, are denoted $\mathcal{O}_{e m, k}, \mathcal{O}_{e m, R}$, and $\mathcal{O}_{p e}$, respectively.

All three observers use the same model equations, except for which fault that is to be estimated, and the model equations are given by (22). As stated above, the single fault assumption is used leading to that two faults in (22) are assumed to be zero in the observers, and $x_{1}$ in (21a) only includes the fault that is to be estimated in the observer. The observer used to estimate the fault $f_{e m, k}$ is e.g.

$$
\mathcal{O}_{f e m, k}:\left\{\begin{array}{l}
\hat{f}_{e m, k, t+1}=\hat{f}_{e m, k, t}+K_{t}\left(y_{t}-\hat{y}_{t}\right) \\
\hat{y}_{t}=h\left(\left[\begin{array}{c}
\hat{f}_{e m, k, t} \\
0 \\
0
\end{array}\right], G\left(\left[\begin{array}{c}
\hat{f}_{e m, k, t} \\
0 \\
0
\end{array}\right], u_{t}\right), u_{t}\right)
\end{array}\right.
$$

Note that it is only one of the three observers that ideally estimates a correct value of the fault. For example, when $f_{e m, k}$ has occurred, $\hat{f}_{e m, R}$ and $\hat{f}_{p e}$ estimated in $\mathcal{O}_{e m, R}$ and $\mathcal{O}_{p e}$ respectively, can take a value separated from zero even though the actual faults $f_{e m, R}=f_{p e}=0$.

The faults are induced one by one in the vehicle model to evaluate the designed observers. The estimated faults using the three observers when $\left\{f_{e m, k}, f_{e m, R}, f_{p e}\right\}=\{-0.03,0,0\}$ are shown in Figure 4. In the upper plot, $\hat{f}_{e m, k}$ is shown and it can be seen that the fault is accurately estimated using $\mathcal{O}_{e m, k}$. The lower two plots present $\hat{f}_{e m, R}$ and $\hat{f}_{p e}$, and since the induced fault in the simulation is $f_{e m, k}$ and the three faults affect the model (22) differently, $\hat{f}_{e m, R}$ and $\hat{f}_{p e}$ do not converge to constant values. The reason is that $\hat{f}_{e m, R}$ and $\hat{f}_{p e}$ varies with the operating point of the machine to achieve the same outputs 

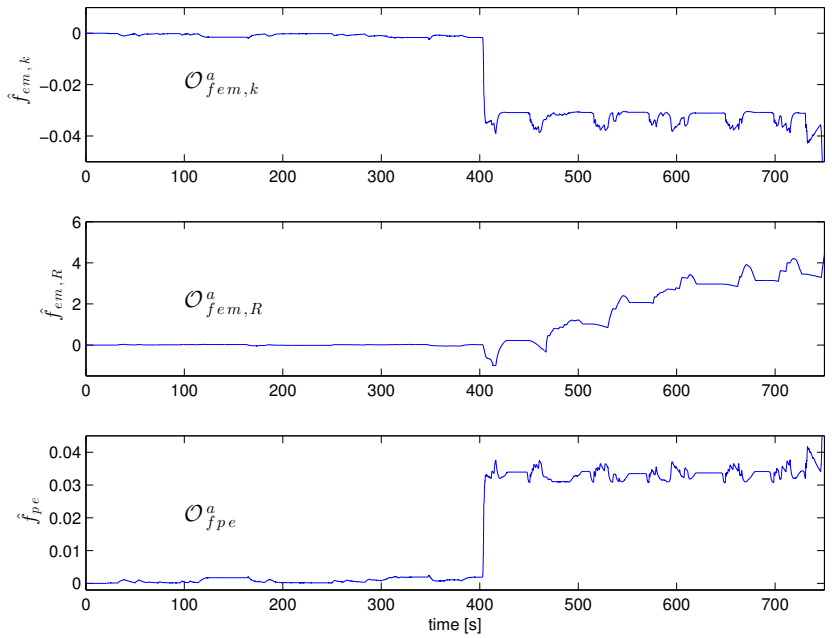

Fig. 5. The estimated faults in the three observers based on the analytical model when $f_{e m, k}=-0.03$.

of the system as the measurements do. The information about what estimated faults that converge to constant values will later be used in the diagnosis system design to pinpoint what fault that has occurred. The results when inducing any of $f_{e m, R}$ and $f_{p e}$ in the vehicle model are similar to the results in Figure 4 when $f_{e m, k}$ is induced.

Note that the computational complexity of the three single fault observers is lower compared the single observer estimating all three faults simultaneously.

2) Three observers based on only the analytical model: In the above designed observers the combined model (22) is used. To investigate how the modeling error in the analytical model, see Figure 1, affect the diagnostic performance, three observers are designed based on the analytical model. The three observers, denoted by $\mathcal{O}_{e m, k}^{a}, \mathcal{O}_{e m, R}^{a}$, and $\mathcal{O}_{p e}^{a}$, are designed using the same principle as in Section V-B1, and the inputs $u$ and outputs $y$ are the same as in (23) and (24). The algebraic variables are denoted by $x_{2}^{a}$, and are computed from $G^{a}\left(x_{1}, u\right)$ given by (5), (6), (9), (12), and (16) as

$$
\underbrace{\left[\begin{array}{l}
k \\
R_{e m} \\
U_{e m}^{c t r l} \\
U_{e m} \\
I_{e m} \\
T_{e m}^{a} \\
I_{b}
\end{array}\right]}_{x_{2}^{a}}=\underbrace{\left[\begin{array}{l}
k^{n o m}\left(1+f_{e m, k}\right) \\
R_{e m}^{n o m}\left(1+f_{e m, R}\right) \\
\frac{R_{e m}^{n m}}{k^{n o m}}\left(T_{e m, r e q}+c_{f} \omega_{e m}\right)+k^{n o m} \omega_{e m} \\
U_{e m}^{c t r l}\left(1+f_{p e}\right) \\
\frac{1}{R_{e m}}\left(U_{e m}-k \omega_{e m}\right) \\
k I_{e m}-c_{f} \omega_{e m} \\
\frac{U_{e m} I_{e m}}{U_{b}}
\end{array}\right]}_{G^{a}\left(x_{1}, u\right)}
$$

The same fault case as in Section V-B1 is considered, i.e. $f_{e m, k}=-0.03$, and the estimated faults using (29) are shown in Figure 5. By comparing Figures 4 and 5 it is clear that the different models used in the observers results in different values of the estimated faults. Note especially that the fault that is induced, $f_{e m, k}$, is more accurately estimated in $\mathcal{O}_{f e m, k}$ compared to $\mathcal{O}_{f e m, k}^{a}$, see the upper plots in Figures 4 and 5. The reason is that the analytical model (29) does not describe the outputs $T_{e m}$ and $I_{b}$ as good as the combined model (22).
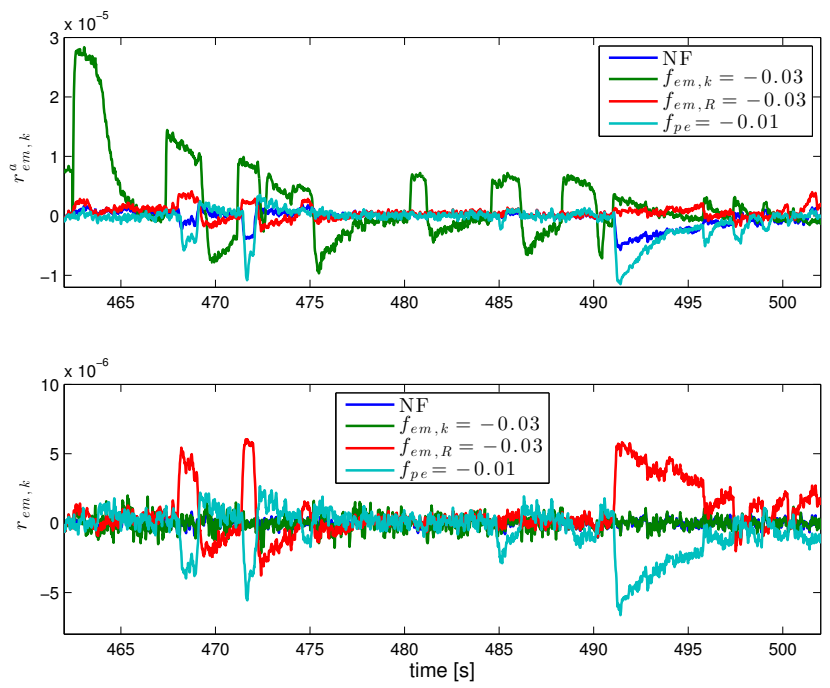

Fig. 6. Residuals based on $\hat{f}_{e m, k}$ computed by $\mathcal{O}_{e m, k}^{a}$ in the upper plot and $\mathcal{O}_{e m, k}$ in the lower plot for the faulty and non-faulty cases.

\section{Residual generators and test quantities for fault isolation}

When an estimated fault is nonzero the system is assumed faulty, and it is clear from Figures 4 and 5 that the estimated faults becomes nonzero fast after the fault is induced in the model and the faults are thereby easily detected. However, to pinpoint what fault that has occurred is more difficult since the faults can not be accurately estimated using one observer, $\mathcal{O}_{\text {all }}$. The three observers $\mathcal{O}_{e m, k}, \mathcal{O}_{e m, R}$, and $\mathcal{O}_{p e}$ are now used to generate residuals and test quantities for fault isolation.

1) Residual generators and test quantity formation: The faults affect the system to be monitored in different ways, see (22) and (29), which in combination with the constant fault assumption is used to isolate the faults. The basic idea is that with an observer dedicated to estimate fault $f_{i}$, the estimate $\hat{f}_{i}$ will converge to a constant if $f_{i}$ is the true fault. However, if $f_{j} \neq f_{i}$ is the true fault, the estimate $\hat{f}_{i}$ will in general not converge to a constant, i.e. $\hat{f}_{i, t} \neq \hat{f}_{i, t-1}$. For each observer, a residual is generated based on the change in the estimated faults in the observers between two time steps as

$$
r_{t}=\hat{f}_{i, t}-\hat{f}_{i, t-1}
$$

The residual generator based on $\hat{f}_{e m, k}$ estimated in $\mathcal{O}_{e m, k}$ is denoted as $r_{e m, k}$, and this signal is presented in the lower plot in Figure 6 for the different faults induced in the vehicle. As can be seen, $r_{e m, k}$ reacts in a similar way in the fault free case and when $f_{e m, k}=-0.03$. When any of the faults $f_{e m, R}$ and $f_{p e}$ occurs the signal is clearly separated from the fault free case, and therefore the residual has reacted as expected.

In Section V-B it is stated that the faults are more accurately estimated using the observers $\mathcal{O}_{e m, k}, \mathcal{O}_{e m, R}$, and $\mathcal{O}_{p e}$, compared to $\mathcal{O}_{e m, k}^{a}, \mathcal{O}_{e m, R}^{a}$, and $\mathcal{O}_{p e}^{a}$, see Figures 4 and 5 . To evaluate the influence of this in the diagnosis system, the corresponding residual to $r_{e m, k}$ based on $\mathcal{O}_{e m, k}^{a}$ is shown in the upper plot in Figure 6, and is denoted as $r_{e m, k}^{a}$. This residual is not expected to react to the fault $f_{e m, k}$, but as can be seen in the figure, the residual is significantly separated from the 
TABLE I

DECISION STRUCTURE FOR THE DESIGNED DIAGNOSIS SYSTEM.

\begin{tabular}{c|ccc} 
& $f_{\text {em }, k}$ & $f_{\text {em }, R}$ & $f_{p e}$ \\
\hline$T 1$ & & $\mathrm{X}$ & $\mathrm{X}$ \\
$T 2$ & $\mathrm{X}$ & & $\mathrm{X}$ \\
$T 3$ & $\mathrm{X}$ & $\mathrm{X}$ & \\
\hline
\end{tabular}

fault free case when $f_{e m, k}=-0.03$. To achieve a correct diagnostic decision there is a need to investigate what causes this separation, and to compensate for the difference in the residual. The results are the same for all three residuals based on the analytical model, and therefore it is more time consuming to design a diagnosis system based on $\mathcal{O}_{e m, k}^{a}, \mathcal{O}_{e m, R}^{a}$, and $\mathcal{O}_{p e}^{a}$, compared to $\mathcal{O}_{e m, k}, \mathcal{O}_{e m, R}$, and $\mathcal{O}_{p e}$. Due to this result, the residuals used in the further diagnosis system design process are based on the combined map and analytical models.

To reduce the impact of noise in the residuals in the decision making, the residuals are post processed to form test quantities. There are many approaches available. Here the CUSUM algorithm is used [21], [22]

$$
T_{t}=\max \left\{0, T_{t-1}+\left|r_{t}\right|-\nu\right\}
$$

where $\nu$ is a design parameter that corresponds to the noise level in the residuals. To evaluate the performance, normalized test quantities, $T_{\text {norm }}$, are calculated based on the maximum value, $T_{\max }^{N F}$, of $T$ in the fault free case. An alarm is generated when $T_{\text {norm }}>1$ and the design parameter $\Phi \in[0,1]$ states the margin to false alarm, and is here set to 0.5

$$
T_{\text {norm }}=\frac{T}{T_{\max }^{N F}} \Phi
$$

The decision structure in Table I describes what faults the different tests are expected to react to. Full structural fault isolability is achieved since a unique set of tests ideally react for each fault. The tests $T_{1}, T_{2}$, and $T_{3}$ are based on $\mathcal{O}_{e m, k}$, $\mathcal{O}_{e m, R}$, and $\mathcal{O}_{p e}$ respectively.

Figure 7 presents the normalized test quantities based on $\mathcal{O}_{e m, k}, \mathcal{O}_{e m, R}$, and $\mathcal{O}_{p e}$, when $f_{e m, k}$ is induced as a step in the vehicle model after 400 seconds. According to Table I $T_{2}$ and $T_{3}$ are expected to react, but as can be seen in the upper plot also $T_{1}$ reacts since $T_{\text {norm }}>1$ when the fault is induced. When a fault occurs in the system, the constant fault assumption is not valid. This may lead to that a residual that should not react to the fault according to Table I becomes non-zero, and the corresponding test quantity reacts. To avoid this behavior the time for the fault estimate to converge after the fault occurrence is identified and during this time period the test quantity is not updated.

2) No test quantity update during transients: The start and end times of the time period where the test quantities are not updated is denoted as $t_{u, s}$ and $t_{u, e}$ respectively, i.e. the time interval is $\tau_{u}=\left[t_{u, s}, t_{u, e}\right]$. Let $\tau_{r}$ be the set of time points where the considered residual computed as in (30) changes sign. To find the times $t_{u, s}$ and $t_{u, e}$, the first time the magnitude of the estimated fault is above a threshold $J_{f}$ is used

$$
t_{u, f}=\min \left\{t:\left|\hat{f}_{i, t}\right|>J_{f}\right\}
$$
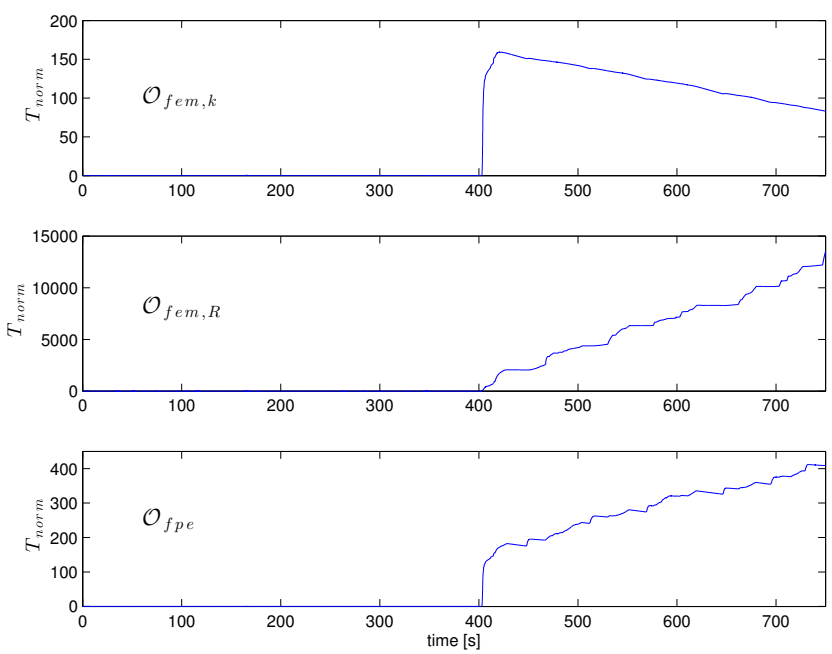

Fig. 7. Test quantities based on the estimated faults presented in Figure 4 when $f_{e m, k}=-0.03$.

After fault occurrence, depending on the fault sign, the residual has a has a positive or negative transient until the estimate has converged. At convergence, the estimate is a constant and the residual changes sign due to noise and uncertainties. Therefore, convergence is detected when the residual start making sign changes again after the initial transient. However, when the operating point of the electric machine results in low fault excitation, the estimated fault does not change much in time even though there is a difference in the real fault and the estimated fault. This results in that the residual may change sign due to noise and uncertainties, even though the estimated fault has not reached the final value when the fault is induced. The magnitude of the electric machine torque is closely related to the fault excitation, and all time instances the torque is above a threshold $J_{T_{e m}}$ is defined as

$$
\tau_{e x}=\left\{t:\left|T_{e m, t}\right|>J_{T_{e m}}\right\}
$$

Based on the defined times, $t_{u, s}$ and $t_{u, e}$ are computed by

$$
\begin{aligned}
& t_{u, s}=\max \left\{t: t \in \tau_{e x} \cap \tau_{r}, t<t_{u, f}\right\} \\
& t_{u, e}=\min \left\{t: t \in \tau_{e x} \cap \tau_{r}, t>t_{u, f}\right\}
\end{aligned}
$$

In Figure 8 the estimated fault $\hat{f}_{e m, k}$ is shown, as well as the corresponding residual and test quantities. In the figure the times $t_{u, f}, t_{u, s}$, and $t_{u, e}$ are also shown. Figure 9 shows the test quantities when these are not updated in the time interval $\tau_{u}$. The upper plot shows that there is no false alarm when the fault is induced in the system, and the other two tests react to the fault as expected. The reaction time for these tests is longer compared to when updating the test quantities at all times, and how long this time is depends on the excitation of the monitored system. Therefore are the test quantities updated at all times used for fault detection and the test quantities presented in Figure 9 are used for fault isolation.

The induced faults occur as a step, and even though the assumption was that the faults are constant or slowly varying, the diagnosis system accurately detects and isolates these stepfaults, i.e., works well even in this worst case situation. 

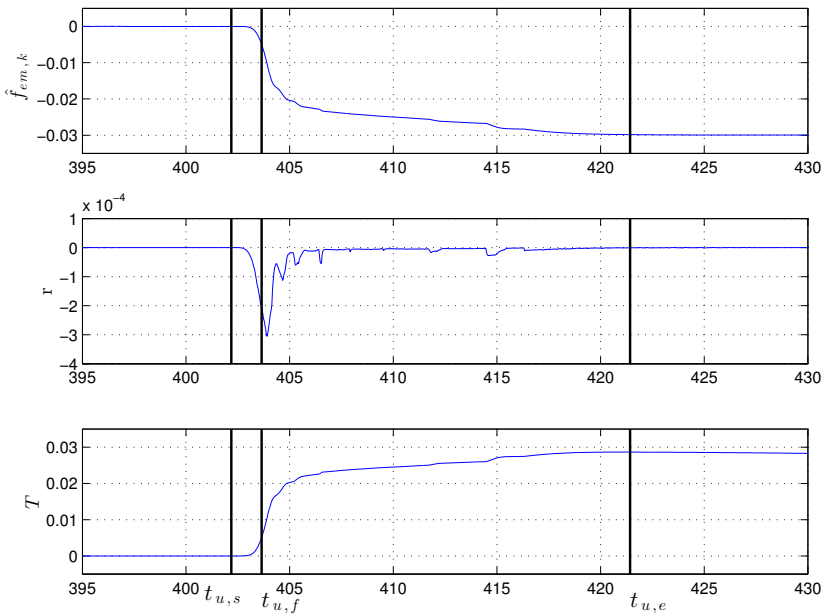

Fig. 8. Illustration of the times used to state when to not update the test quantity exemplified with $\mathcal{O}_{f e m, k}$ when $f_{e m, k}=-0.03$.
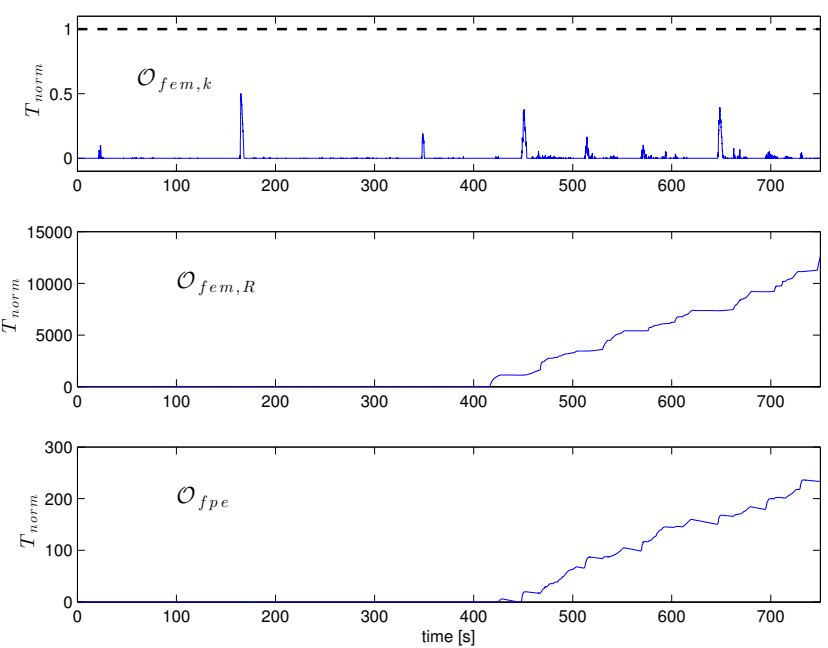

Fig. 9. The test quantities based on the estimated faults in Figure 4, but the test quantities are not updated when the fault is induced in the model.

\section{Summing up}

In the case study the diagnostic performance is demonstrated to be significantly better using the combined model (22) compared to the analytical model (29) in the diagnosis system design, see e.g. Figure 6. Further, a scheme of using several observers estimating one fault each is used to isolate the faults. At the time instant when a fault occurs in the system the fault is obviously not constant, thus violating $\dot{f}=0$, and to avoid false test reaction due to this, an approach by not updating the test quantities is successfully used, compare Figures 7 and 9.

\section{CONCLUSIONS}

Development time is crucial for the total cost, and the main contribution here is a methodology that reduces engineering time for the design of the diagnosis system while still achieving desired fault detection and isolation properties. A key enabling observation is that steps earlier in the development process, e.g. in control and in powertrain management steps, usually develops carefully measured look-up models for the fault free case. The main idea here is to combine these models with analytical fault models that explicitly describe how the faults affect the monitored system. The approach is illustrated in the design of a diagnosis system for the power electronics and the electric machine in an HEV, where an extensive simulation study shows the benefits of the approach. This includes the specific scheme of using several observers estimating one fault each. In summary, the clear improvement in overall performance demonstrates that the method achieves both good fault detectability and isolability performance, without the need for neither measurements of a faulty system nor detailed physical modeling.

\section{REFERENCES}

[1] L. Eriksson and L. Nielsen, Modeling and Control of Engines and Drivelines. John Wiley \& Sons, 2014.

[2] S. J. Qin, "Survey on data-driven industrial process monitoring and diagnosis," Annual Reviews in Control, vol. 36, no. 2, pp. 220 - 234, 2012.

[3] X. Dai and Z. Gao, "From model, signal to knowledge: A data-driven perspective of fault detection and diagnosis," Industrial Informatics, IEEE Transactions on, vol. 9, no. 4, pp. 2226-2238, Nov 2013.

[4] Z. Gao, C. Cecati, and S. Ding, "A survey of fault diagnosis and faulttolerant techniques - part I: Fault diagnosis with model-based and signalbased approaches," Industrial Electronics, IEEE Transactions on, vol. 62, no. 6, pp. 3757-3767, June 2015.

[5] J. de Kleer, A. Mackworth, and R. Reiter, "Characterizing diagnoses and systems," Artificial Intelligence, vol. 56, no. 2-3, pp. 197-222, 1992.

[6] J. de Kleer and B. C. Williams, "Diagnosing multiple faults," Artificial Intelligence, vol. 32, pp. 97-130, April 1987.

[7] P. Struss and O. Dressier, ""physical negation": integrating fault models into the general diagnostic engine," in Proc. of the 11th int. joint conference on AI - Vol 2, San Francisco, 1989, pp. 1318-1323.

[8] E. Chow and A. Willsky, "Analytical redundancy and the design of robust failure detection systems," Automatic Control, IEEE Transactions on, vol. 29 , no. 7, pp. $603-614$, July 1984.

[9] M. Staroswiecki and G. Comtet-Varga, "Analytical redundancy relations for fault detection and isolation in algebraic dynamic systems," Automatica, vol. 37, no. 5, pp. 687 - 699, 2001.

[10] R. Isermann, Fault Diagnosis Systems - An Introduction from fault Detection to Fault Tolerance. Springer Verlag, 2006.

[11] P. M. Frank, "Enhancement of robustness in observer-based fault detection," Int. J. of Control, vol. 59, no. 4, pp. 955-981, 1994.

[12] G. Foo, X. Zhang, and D. Vilathgamuwa, "A sensor fault detection and isolation method in interior permanent-magnet synchronous motor drives based on an extended kalman filter," Industrial Electronics, IEEE Transactions on, vol. 60, no. 8, pp. 3485-3495, Aug 2013.

[13] M. Blanke, M. Kinnaert, J. Lunze, and M. Staroswiecki, Diagnosis and Fault-Tolerant Control, 2nd ed. Springer, 2006.

[14] C. Sundström, E. Frisk, and L. Nielsen, "Fault monitoring of the electric machine in a hybrid vehicle," in 7th IFAC Symposium on Advances in Automotive Control, Tokyo, Japan, 2013, pp. 548-553.

[15] Z. Zhu and D. Howe, "Electrical machines and drives for electric, hybrid, and fuel cell vehicles," Proc. of the IEEE, vol. 95, no. 4, pp. $746-765$, April 2007.

[16] P. Mellor, "High efficiency drive-trains for electric and hybrid vehicles," in IEE Colloquium on Electrical Machine Design for All-Electric and Hybrid-Electric Vehicles, 1999, pp. 8/1 -8/7.

[17] L. Guzzella and A. Sciarretta, Vehicle Populsion System, Introduction to Modelling and Optimization, 2nd ed. Zürich: Springer Verlag, 2007.

[18] G. Zhu, L.-A. Dessaint, O. Akhrif, and A. Kaddouri, "Speed tracking control of a permanent-magnet synchronous motor with state and load torque observer," IEEE Trans. on Industrial Electronics, vol. 47, no. 2, pp. $346-355$, Apr. 2000.

[19] C. Sundström, "Vehicle level diagnosis for hybrid powertrains," Tech. Rep., 2011, liU-TEK-LIC-2011:27, Thesis No. 1488.

[20] T. Kailath, A. H. Sayed, and B. Hassibi, Linear Estimation. Prentice Hall, 2000.

[21] E. Page, "Continuous inspection schemes," Biometrika, vol. 41, pp. 100$115,1954$.

[22] F. Gustafsson, Adaptive filtering and change detection. John Wiley \& Sons, 2000. 